University of New Hampshire

University of New Hampshire Scholars' Repository

Physics Scholarship

Physics

$5-1-2010$

\title{
Galactic cosmic ray radiation hazard in the unusual extended solar minimum between solar cycles 23 and 24
}

Nathan A. Schwadron

Nathan.Schwadron@unh.edu

A. J. Boyd

K. Kozarev

M. Golightly

University of New Hampshire

Harlan E. Spence

University of New Hampshire, harlan.spence@unh.edu

See next page for additional authors

Follow this and additional works at: https://scholars.unh.edu/physics_facpub

Part of the Physics Commons

\section{Recommended Citation}

Schwadron, N. A., A. J. Boyd, K. Kozarev, M. Golightly, H. Spence, L. W. Townsend, and M. Owens(2010), Galactic cosmic ray radiation hazard in the unusual extended solar minimum between solar cycles 23 and 24, Space Weather, 8, S00E04, doi:10.1029/2010SW000567.

This Article is brought to you for free and open access by the Physics at University of New Hampshire Scholars' Repository. It has been accepted for inclusion in Physics Scholarship by an authorized administrator of University of New Hampshire Scholars' Repository. For more information, please contact Scholarly.Communication@unh.edu. 


\section{Authors}

Nathan A. Schwadron, A. J. Boyd, K. Kozarev, M. Golightly, Harlan E. Spence, L. W. Townsend, and M. Owens 


\title{
Galactic cosmic ray radiation hazard in the unusual extended solar minimum between solar cycles 23 and 24
}

\author{
N. A. Schwadron, ${ }^{1}$ A. J. Boyd, ${ }^{1}$ K. Kozarev, ${ }^{1}$ M. Golightly, ${ }^{2}$ H. Spence, ${ }^{2}$ \\ L. W. Townsend, ${ }^{3}$ and M. Owens ${ }^{4}$ \\ Received 1 January 2010; revised 14 March 2010; accepted 25 March 2010; published 22 May 2010.
}

[1] Galactic cosmic rays (GCRs) are extremely difficult to shield against and pose one of the most severe long-term hazards for human exploration of space. The recent solar minimum between solar cycles 23 and 24 shows a prolonged period of reduced solar activity and low interplanetary magnetic field strengths. As a result, the modulation of GCRs is very weak, and the fluxes of GCRs are near their highest levels in the last 25 years in the fall of 2009. Here we explore the dose rates of GCRs in the current prolonged solar minimum and make predictions for the Lunar Reconnaissance Orbiter (LRO) Cosmic Ray Telescope for the Effects of Radiation (CRaTER), which is now measuring GCRs in the lunar environment. Our results confirm the weak modulation of GCRs leading to the largest dose rates seen in the last 25 years over a prolonged period of little solar activity.

Citation: Schwadron, N. A., A. J. Boyd, K. Kozarev, M. Golightly, H. Spence, L. W. Townsend, and M. Owens (2010), Galactic cosmic ray radiation hazard in the unusual extended solar minimum between solar cycles 23 and 24, Space Weather, 8, S00E04, doi:10.1029/2010SW000567.

\section{Background: Galactic Cosmic Rays and Human Health}

[2] In balloon flights in 1912-1913, Hess [1912, 1913] first measured a form of radiation that intensified with altitude. Millikan and Bowen [1926] realized that this radiation couldn't come from the Sun since its intensity didn't vary from day to night, and thus called it "Cosmic Ray" radiation. The majority of this radiation is from galactic comic rays (GCRs), which are accelerated outside our Solar System, most likely by the strong shocks that result from supernova explosions in our galaxy. GCRs have enormous energies (100 MeV to $10 \mathrm{GeV}$ ) and near-light speeds. They continually bombard Earth's atmosphere, producing secondary particles and radiation through cascading highenergy collisions.

[3] Because most GCR radiation that penetrates into the near-Earth space environment is shielded by Earth's magnetic field, it does not pose a common health hazard.

\footnotetext{
${ }^{1}$ Department of Astronomy, Boston University, Boston, Massachusetts, USA.

${ }^{2}$ Institute for the Study of Earth, Oceans, and Space, University of New Hampshire, Durham, New Hampshire, USA.

${ }^{3}$ Department of Nuclear Engineering, University of Tennessee, Knoxville, Tennessee, USA.

${ }^{4}$ Space Environment Physics Group, Department of Meteorology, University of Reading, Reading, UK.
}

However, during space travel, GCRs are difficult to shield [Wilson et al., 1991; Cucinotta et al., 2001a, 2001b] since they produce secondary radiation in shielding and other material that is even more hazardous than the GCRs themselves. On long duration missions, GCR radiation is the primary health hazard to astronauts [Space Science Board, 1973; Space Studies Board, 1996; Cucinotta et al., 2001a].

[4] In the outer heliosphere, beyond 100 AU, the slowing of solar wind is thought to form a large magnetic barrier that shields out $>90 \%$ of the GCR radiation present in interstellar space [Florinski et al., 2003] at energies below $\sim 100 \mathrm{MeV}$. Because this reduction is so large, even a very small change in the shielding efficiency can have a large impact on the radiation environment in the solar system. However, because these regions have never been directly sampled or observed, there is great uncertainty about the physics of outer heliospheric shielding and its sensitivity to changes in the solar wind output and the local interstellar medium. A small fraction of GCRs penetrate into the heliosphere and propagate toward the Sun and planets. These residual GCRs are modulated by the solar wind's magnetic field in the inner heliosphere.

[5] Forbush [1954] discovered the 11 year modulation of GCRs, which is explained by the transport of GCRs through heliospheric magnetic fields [Parker, 1965; Gleeson and Axford, 1968; Urch and Gleeson, 1972]. During periods of high solar activity (solar maximum), which occur each 
11 years, the heliospheric magnetic field magnitudes increase due to a much larger rate of coronal mass ejections (CMEs) from the Sun. As a result, the solar wind's intense solar maximum magnetic field is more effective at sweeping GCRs out of the inner heliosphere, which causes a strong reduction of the GCR flux. A 22 year cycle is also evident: GCR flux maxima are typically sharply peaked for even numbered solar minima (when the large scale solar magnetic field is inward in the north; $A<0$ ), whereas odd numbered solar minima (when the solar magnetic field is outward in the north; $\mathrm{A}>0$ ) show more broadly peaked GCR flux maxima. Over the 22 year solar magnetic cycle, known as the Hale cycle, the large-scale solar magnetic field reverses its polarity over the first solar activity cycle ( 11 years) and then returns to its original polarity in the next solar activity cycle. Most of the actual magnetic reversal happens over a 2-4 year period centered on solar maximum and is thought to be forced by the release of CMEs [Owens et al., 2007; Schwadron et al., 2008]. The solar magnetic reversal has a strong effect on the entry of GCRs into the heliosphere. Positively charged cosmic rays drift in from the heliospheric polar regions when the Sun's north polar field is directed outward $(\mathrm{A}>0)$. In contrast, when the Sun's north polar field is directed inward $(\mathrm{A}<0)$, positively charged cosmic rays drift inward along the heliospheric current sheet where they are scattered by irregularities in the current sheet, by coronal mass ejections and other large-scale interaction regions. Conversely, negatively charged cosmic rays (electrons) drift inward from directions opposite to the positively charged cosmic rays. Potgieter and le Roux [1992] and Ferreira and Potgieter [2004] approximately reproduce the $\sim 22$ year cycle in the GCR fluxes using a time-dependent modulation model, which includes gradient, curvature, and current sheet drift effects [Jokipii et al., 1977] and diffusion parallel and perpendicular to the magnetic field.

[6] Large changes in the Local Interstellar Medium (LISM) have dramatic effects on the heliosphere and the radiation environment of the solar system. For example, a typical enhancement in the density of the local interstellar medium by a factor of 10 causes the entire heliosphere to shrink to about a quarter of its current size [Zank and Frisch, 1999], and increases the fluxes of GCRs at Earth by a factor of 2-6 [Scherer et al., 2002]. Such large changes in the LISM have certainly occurred in the past and will occur again in the future [Zank and Frisch, 1999].

[7] We do not currently have the observational knowledge required to understand how the local interstellar medium interacts with the heliosphere; continued observations such as those provided by the Interstellar Boundary Explorer mission (IBEX) [McComas et al., 2009] and the Voyager satellites [e.g., Stone et al., 2005; Richardson et al., 2008] are important for better understanding the radiation environment that must be traversed by astronauts for long missions to distant destinations such as Mars.

[8] On Earth, radioisotope ${ }^{10} \mathrm{Be}$ provides a recent record of cosmic ray fluxes. The isotope is produced in Earth's upper atmosphere by spallation reactions of cosmic rays
(CR) protons with energies higher than about $100 \mathrm{MeV}$ and secondary neutrons with atmospheric nitrogen and oxygen [Masarik and Beer, 1999]. ${ }^{10} \mathrm{Be}$ records in Antarctic ice show two prominent peaks 35,000 and 60,000 years ago, when the radioisotope production rate was about twice the current value for about 1500 and 2000 years, respectively [Raisbeck et al., 1987]. The 35,000 year peak is also present in marine sediment records [Cini Castagnoli et al., 1995; McHargue et al., 1995]. The fact that the ${ }^{10} \mathrm{Be}$ radioisotope changes significantly with time shows that significant changes in cosmic ray fluxes have occurred in the past. But the cause for these changes remains controversial. The most accepted explanation is that the peaks are caused by geomagnetic magnetic field excursions, which is supported by the observed correlation between geomagnetic field strength and ${ }^{10}$ Be levels in marine sediment records [McHargue et al., 2000; Wagner et al., 2000]. However, this explanation would suggest that the ${ }^{10}$ Be enhancements should be uniform over the globe, which contradicts the large variations observed at high latitudes where geomagnetic effects are small. Further, Cini Castagnoli et al. [1998] found that the ${ }^{10} \mathrm{Be}$ level starts to increase some 2000 years before the drop in Earth's magnetic field, as reflected by the magnetization measured in the same core sample. Thus, many of the long-term changes in the cosmic ray fluxes incident on Earth may be due to effects external to the Earth; either due to changes in the shielding of the inner heliosheath or due to changes in the incident fluxes of GCRs from outside the heliosphere.

[9] Could the GCR fluxes in the heliosphere change rapidly in the future due to rapidly changing conditions in the LISM? For the period from $1700 \mathrm{AD}$ to present-day, the ${ }^{10} \mathrm{Be}$ record from ice cores can be compared with sunspot data [Beer et al., 1990]. The result is a remarkably steady anticorrelation, which demonstrates how changes in the solar wind's magnetic field over the solar cycle affect GCRs in the inner solar system. As described above, higher solar activity (when sunspot numbers are large) is correlated with increased interplanetary magnetic field strength, which, in turn, reduces the number of GCRs entering the inner heliosphere. A further implication of the strong and remarkably steady anticorrelation between sunspot number and GCR flux is that, over the last 300 years, the incident flux of GCRs on the heliosphere, and the shielding of GCRs in the inner heliosheath have been relatively constant.

[10] The primary question taken up in this paper is the dose rates induced by GCRs. The GCR environmental model of Badhwar and O'Neill [1991, 1992, 1993, 1994, 1996] is used to model the species-dependent GCR flux over time. The Badhwar-O'Neill model is based on the fitting of measured differential energy spectra to stationary solutions of the Fokker-Planck equation parameterized by an assumed form for the diffusion coefficient that varies as a function of the interplanetary modulation potential (measured in MV). The High-charge (Z) and Energy (HZE) Transport code (HZETRN) is solved using a onedimensional, analytical formulation of the Boltzmann 


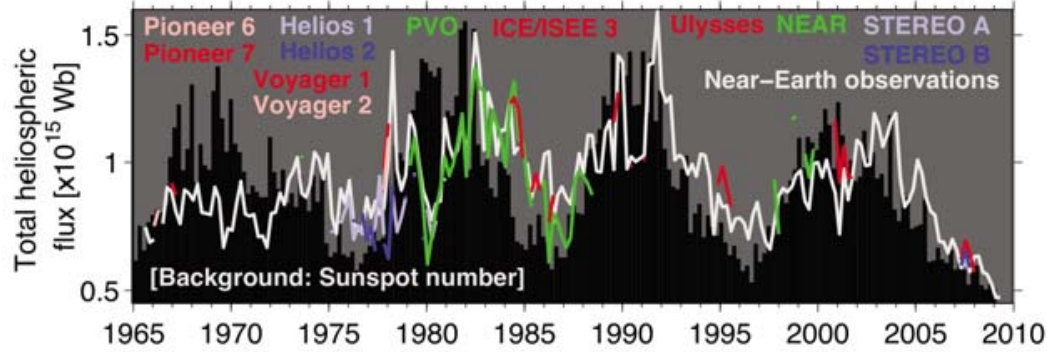

Figure 1. Total heliospheric magnetic flux estimated from in situ spacecraft observations over four complete solar cycles. Sunspot number is shown as the black background. There is an approximate doubling of heliospheric flux from solar minimum to maximum (which may be the result of closed flux added to the heliosphere by coronal mass ejections). The current minimum is exhibiting the lowest flux yet observed. Plot adapted from Owens et al. [2008].

transport equation to describe the transport of GCRs through shielding and tissue. The effect of the long-range Coulomb force and electron interaction is treated as a continuous slowing-down process. Atomic (electronic) stopping power coefficients with energies above a few $\mathrm{MeV} /$ nucleon are calculated using Bethe's theory including Bragg's rule, Ziegler's shell corrections, and effective charge. Nuclear reaction cross sections are obtained from curve fits to quantum scattering calculations and total cross sections are obtained with a Ramsauer formalism. Nuclear fragmentation cross sections are calculated with a semiempirical abrasion-ablation fragmentation model, NUCFRG2, also developed at NASA Langley. The code transports heavy charged particles $(\mathrm{Z}=1$ through 26$)$ in the incident GCR spectrum, and all of their reaction products, including secondary neutrons, through as many as 5 layers of shield materials and water, which is used as a surrogate for human tissue. The output from the code includes particle fluxes or fluences for all heavy charged particles and neutrons, absorbed dose, dose equivalents, and linear energy transfer (LET) spectra in water for any desired location in any of the shield materials.

[11] A 3-layer version of HZETRN 2005 has been configured for the Earth-Moon-Mars Radiation Environment Module (EMMREM) to calculate GCR dose and dose equivalent (L. Townsend et al., Transmission of solar energetic particles and galactic cosmic rays through the Mars atmosphere, submitted to Space Weather, 2009). The code has been used to develop a look-up table of daily effective dose, organ doses and dose equivalents behind varying thicknesses of aluminum shielding. GCR flux input into the calculations is taken from the BadhwarO'Neill GCR model using modulation potentials ranging from the most highly probable solar minimum (400 MV) to solar maximum conditions (1800 MV) in the solar cycle. This model is the standard one used for space operations at the Space Radiation Analysis Group (SRAG) at NASA Johnson Space Center. A look-up table is used because the large spread in interplanetary magnetic field conditions, large numbers of GCR ion species and their many reaction product secondary particles must be transported through as much as $500 \mathrm{~g} / \mathrm{cm}^{2}$ of shield materials. Calculations of such complex spectra at such depths take approximately half a day for each possible spectrum and cannot be carried out in near real time simulations. Since GCR intensities change very little from day to day, daily dose and dose equivalent estimates are sufficient.

\section{Changes in GCRs in an Extended Solar Minimum (Cycles 23 to 24)}

[12] The solar minimum between cycles 23 and 24 is proving to be extremely interesting. The Sun is remarkably quiet. Recent observations have found that the strength of the interplanetary magnetic field [Owens et al., 2008] has been falling off to new low levels observed during the space age. Figure 1 shows the evolution of the interplanetary magnetic field strength. The precipitous recent drop in the observed magnetic field strength is seen at multiple spacecraft and appears to be related to a global reduction in the magnetic flux of the heliosphere.

[13] We show in Figure 2 the modulation potential derived from neutron fluxes at Earth and cosmic rays observed at ACE [O'Neill, 2006]. The modulation potential is derived from the modulation parameter,

$$
\phi(r)=\int_{r}^{R_{b}} \frac{V(x)}{3 \kappa_{1}(x)} d x
$$

where the modulation potential is $\Phi=\mid Z$ el $\phi(r)$. The integral in (1) extends from the inner boundary at radius $r$ to the outer modulation boundary $R_{b}$, the solar wind speed is $V(x)$ and $\kappa_{1}(x)$ is related to the radial diffusion coefficient, $\kappa$. In particular, the form for $\kappa$ is based on a fit to the observed spectrum over time and species [O'Neill, 2006]: $\kappa=\kappa_{1}(\mathrm{r}) P \beta$ where $P$ is the rigidity in GV, $\beta$ is the particle speed over the speed of light, $\kappa_{1}(r) \propto 1+\left(r / r_{0}\right)^{2}$ and $\mathrm{r}_{0}=4 \mathrm{AU}$. In the Badhwar-O'Neill model, reductions in the modulation potential are caused by enhanced diffusion, allowing greater access and therefore higher fluxes of GCRs in the inner heliosphere. 


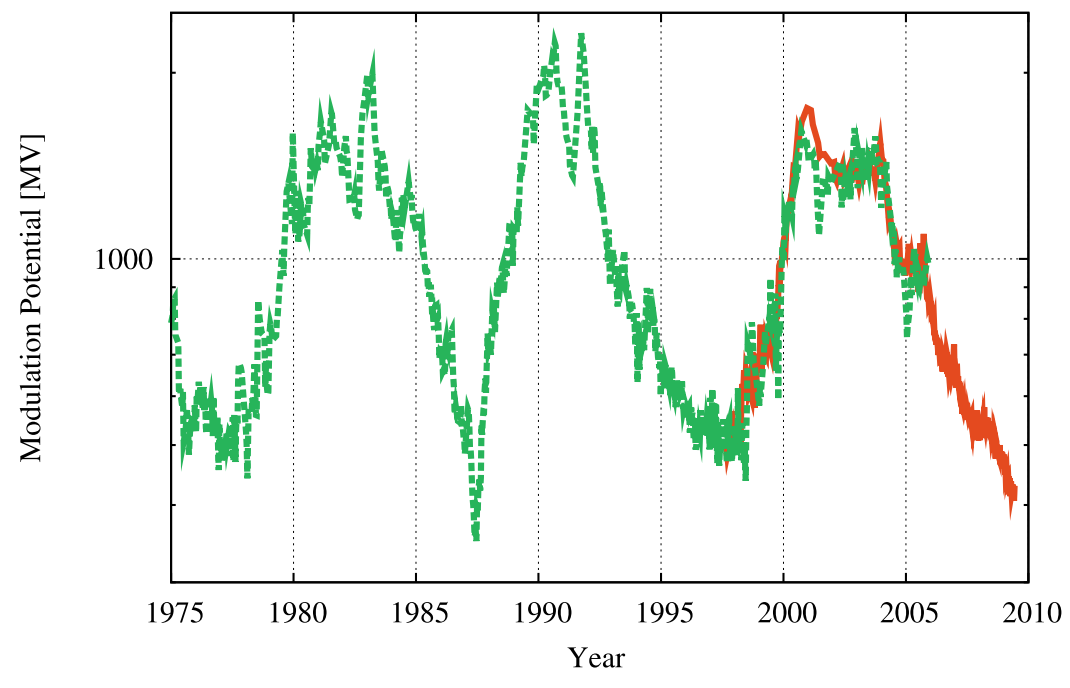

ACE Neutron Data $=\ldots+\cdots=$

Figure 2. The modulation parameter based on observations of neutrons using Climax data and on the Advanced Composition Explorer (ACE) Cosmic Ray Isotope Spectrometer (CRIS) measurements [O'Neill, 2006].

[14] Saganti et al. [2006] show a comparison between the Badhwar-O'Neill model with HZETRN and MARIE Mars observations. They find $21.2 \mathrm{mrad} /$ day predicted by the model versus $21.4 \mathrm{mrad} /$ day measured observed by MARIE. Thus, the combination of HZETRN and the Badhwar-O'Neill model provides an accurate determination of dose and dose rate. The predictions that follow will eventually be compared to measurements from the Lunar Reconnaissance Orbiter (LRO) Cosmic Ray Telescope for the Effects of Radiation (CRaTER).

[15] Figure 3 shows the dose rates to the skin, central nervous system (CNS), blood forming organs (BFO) and the lens using the combination of HZETRN and the Badhwar-O'Neill model for a variety of different shielding levels from $0.3 \mathrm{~g} / \mathrm{cm}^{2}$ to $100 \mathrm{~g} / \mathrm{cm}^{2}$. These doses are calculated in free space where surfaces are exposed over $4 \pi$ sr.

[16] Figure 4 shows the results for the dose-equivalent rates, which take into account quality factors and relative biological effectiveness for the different species composing galactic cosmic rays. The dose-equivalent rates are substantial. For example, behind $0.3 \mathrm{~g} / \mathrm{cm}^{2} \mathrm{Al}$ we find dose rates between 25 and $35 \mathrm{cSv} /$ year, which approach dose limits of $52 \mathrm{cSv}$ (for a 25 year old male), $37 \mathrm{cSv}$ (for a 25 year old female), $72 \mathrm{cSv}$ (for a 35 year old male), and $55 \mathrm{cSv}$ (35 year old female) for 1 year missions and average life loss for an exposure-induced death for radiation carcinogenesis [NASA Technical Standard, 2007, Table 3].

[17] The LRO/CRaTER instrument, which was launched in June 2009, measures the LET spectrum in lunar orbit as an aid in determining risks to human crews on future lunar missions. LET, often a nonlinear function, is defined as the instantaneous energy deposited into the local environment by a given charged particle per unit of distance. As the particle's energy decreases, so does its LET.
The LET spectra allow us to understand the contributions to doses from different particles in the incident spectrum. Part of the preparations for the mission involved estimating the LET spectrum for the anticipated environment that the instrument is likely to see during the one year operational phase of the LRO mission.

[18] Townsend et al. [2010] prepared detailed estimates of LET spectra in the six silicon detectors and two tissueequivalent plastic segments were made using the beta version of the High Energy Transport Code for Human Exploration and Development in Space (HETC-HEDS) Monte Carlo transport code. Tables of LET in each detector component, for incident particle elemental species from hydrogen through iron, were carried out at incident particle energies from $20 \mathrm{MeV} /$ nucleon to $3 \mathrm{GeV} /$ nucleon. The LET values in these tables were parameterized by elemental species and energy for ease in quickly and accurately estimating the LET response for any input solar or GCR spectrum likely to be encountered during the lifetime of the instrument. The parameterized LET values are in excellent agreement with the HETC-HEDS calculations. Typical differences are on the order of a few percent.

[19] Figure 5 shows a comparison between the predictions of dose rate based on the Townsend et al. [2010] tables and predictions using the HZETRN model. Both predictions use GCR fluxes based on the Badhwar-O'Neill model. In the case of the predictions from the HZETRN model, we consider the lens dose behind $0.22 \mathrm{~g} / \mathrm{cm}^{2}$ of $\mathrm{Al}$ shielding exposed to $1.8 \pi \mathrm{sr}$, which is appropriate for the CRaTER D1 detector. The predictions in Figure 5 will provide useful benchmarks for comparison to CRaTER observations.

[20] Figure 6 shows the LET spectra determined by the SPENVIS and CREME-96 models for ions penetrating Si 

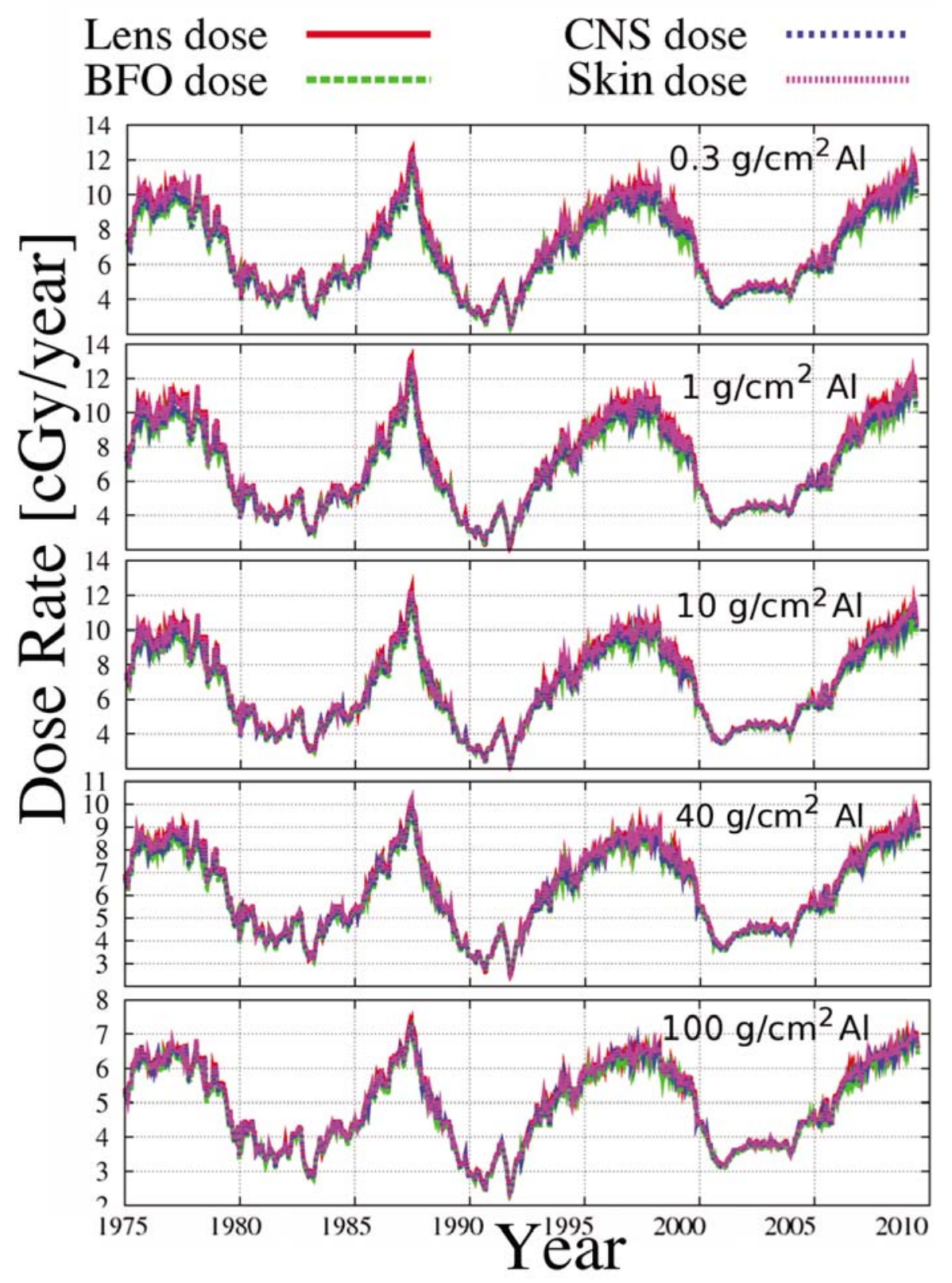

Figure 3. Dose rates from GCRs in free space behind different levels of $\mathrm{Al}$ shielding ranging from 0.3 to $100 \mathrm{~g} / \mathrm{cm}^{2}$. These predictions have been made using a 3-layer version of HZETRN 2005, which has been configured to calculate GCR dose and dose equivalents (Townsend et al., submitted manuscript, 2009) based on GCR fluxes from Badhwar-O'Neill GCR model. We have calculated doses to the lens, central nervous system, blood forming organs, and skin. 


\section{Lens dose $\quad$ CNS dose $\quad \cdots . . . . . . .$.

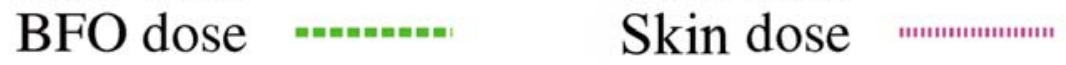

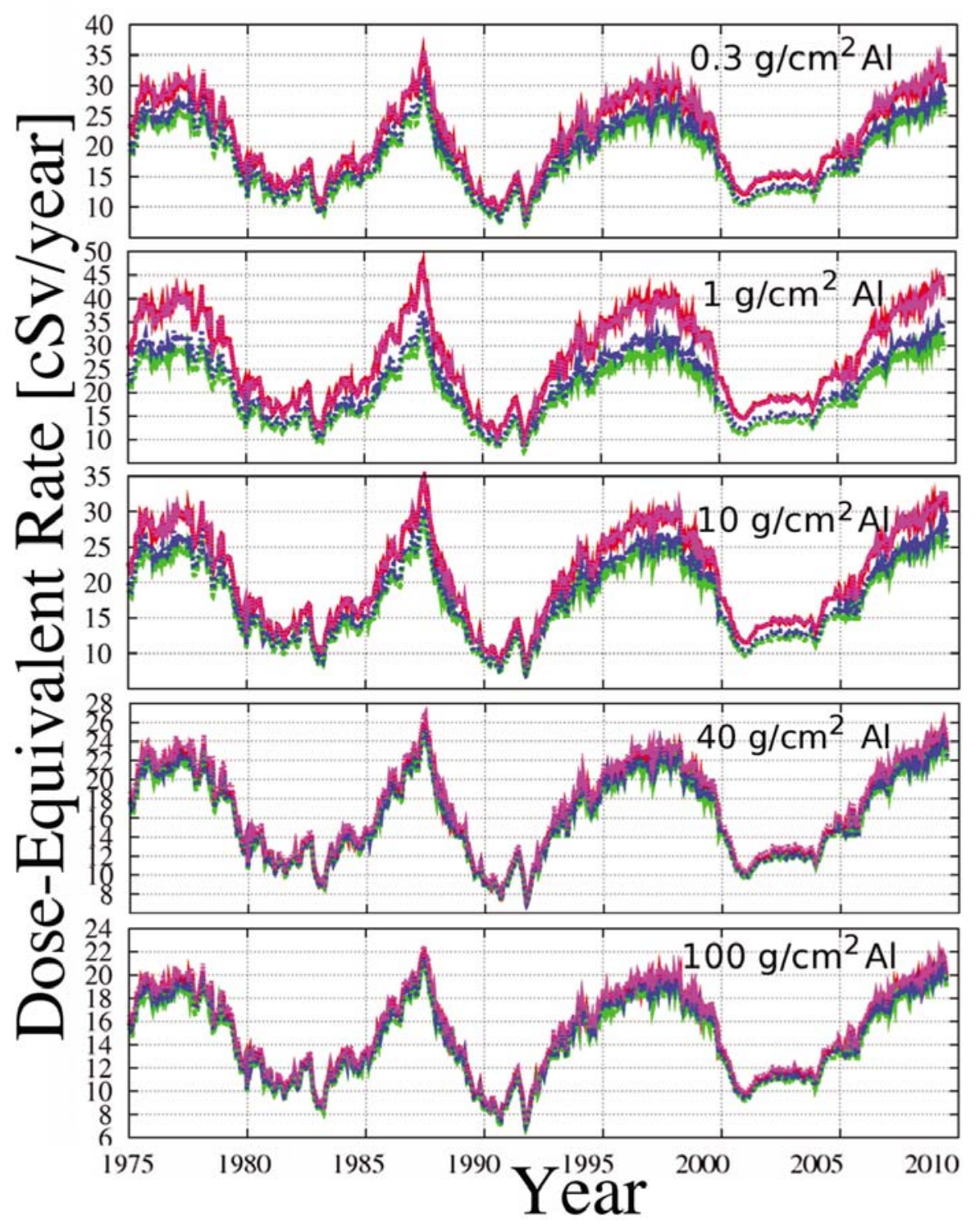

Figure 4. Dose equivalent rates from GCRs in free space behind different levels of Al shielding ranging from 0.3 to $100 \mathrm{~g} / \mathrm{cm}^{2}$. These predictions are similar to Figure 3 but also include quality factors and relative biological effectiveness factors. 


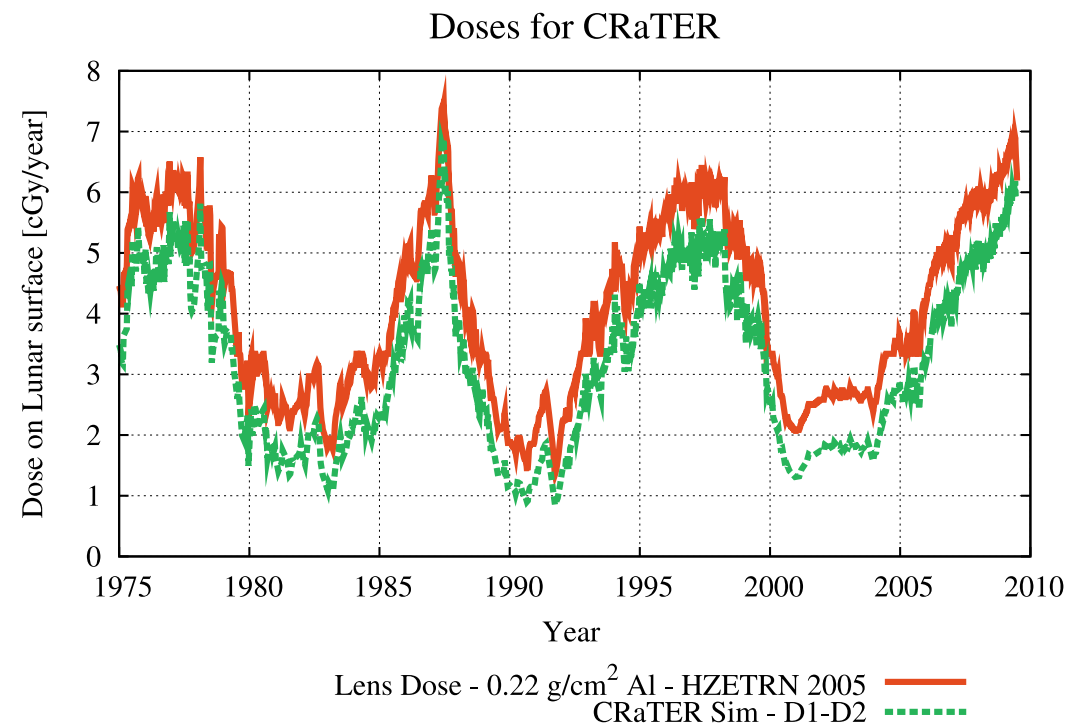

Figure 5. Predictions of dose rates near the lunar surface for the LRO/CRaTER instrument. The red curve shows the lens dose rate computed from HZETRN 2005 model and the Badhwar-O'Neill GCR model. The green curve uses the parameterization of LET and derived dose rates based on the tables of Townsend et al. [2010].

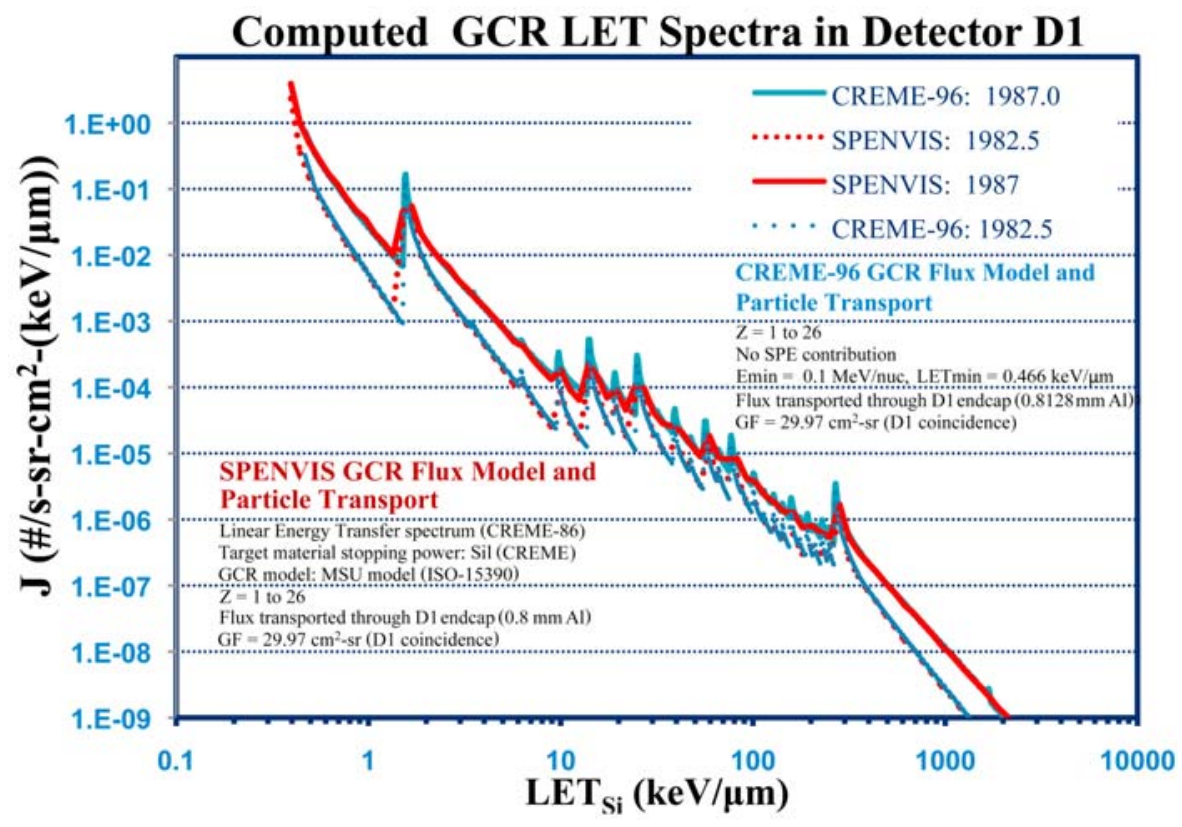

Figure 6. Differential linear energy transfer (LET) spectra determined by the SPENVIS (http:// www.spenvis.oma.be/) and CREME-96 (https://creme96.nrl.navy.mil/) models. LET energy transfer calculations allow us to differentiate the contributions to the dose in terms of species. Low LET contributions arise primarily from protons and alpha particles, whereas higher LET contributions arise from the heavy ions. In this case, we compute LET spectra in Si (through the D1 detector on CRaTER) using atoms with charge $Z=1$ to $Z=26$. The calculations were performed for a solar minimum in 1987 and a solar maximum in 1982.5 . 
(through the D1 detector on CRaTER) with charge $\mathrm{Z}=1$ to 26. The LET spectra show the differences between during solar minimum and solar maximum. These predictions provide a baseline for comparison to LET spectra observed by CRaTER.

\section{Conclusions}

[21] We show predictions and simulations of the radiation effects induced by GCRs. The radiation environment in the extended solar minimum observed in 2009-2010 consists of highly elevated fluxes of GCRs, and accordingly, elevated dose rates. The enhanced dose rates predicted by models are the largest since 1987 and the extended solar minimum has caused an unusually long period when dose rates have been elevated. This result is not surprising considering the reduction in the total heliospheric magnetic flux observed by interplanetary spacecraft (STEREO A and B, Ulysses, ACE, and Wind). Furthermore the reduction in magnetic flux comes during a solar minimum when the heliospheric magnetic field allows better access to the inner heliosphere through gradient and curvature drifts of cosmic rays [Jokipii et al., 1977; Heber et al., 2009]. In a magnetic epoch like the present when the large-scale solar magnetic fields are directed inward in the north (also in the 1960s, 1980s) we expect a more peaked time profile for positively charged GCRs. In contrast, we expect a broadened peak in the time profile of positively charged GCRs in epochs when the large-scale solar magnetic fields are directed outward in the north, as in the 1970s and 1990s. The reduction in heliospheric flux and the GCR drift patterns have caused the extended solar minimum to be both elevated in dose rate and prolonged compared to previous solar minima.

[22] The prolonged solar minimum provides optimal conditions for the measurement of GCRs. We have used models to provide predictions for the CRaTER observations, which will be used in the coming years to test our understanding of radiation effects and interactions.

[23] Thus, we model the dose rates, dose-equivalent rates and LET spectra of galactic cosmic rays in the current prolonged solar minimum and make predictions for the Lunar Reconnaissance Orbiter (LRO) Cosmic Ray Telescope for the Effects of Radiation (CRaTER), which is now measuring GCRs in the lunar environment. Our results show the weak modulation of GCRs causing the largest dose rates seen in the last 25 years over the prolonged solar minimum.

[24] Acknowledgments. Research support from the NASA LWS EMMREM project, grant NNX07AC14G, is gratefully acknowledged.

\section{References}

Badhwar, G. D., and P. M. O'Neill (1991), An improved model of galactic cosmic radiation for space exploration missions, Proc. Int. Conf. Cosmic Rays 22nd, 1, 643-646.
Badhwar, G. D., and P. M. O'Neill (1992), An improved model of galactic cosmic radiation for space exploration missions, $\mathrm{Nucl}$. Tracks Radiat. Meas., 20, 403-410, doi:10.1016/1359-0189(92)90024-P.

Badhwar, G. D., and P. M. O'Neill (1993), Time lag of twenty-two year solar modulation, Proc. Int. Conf. Cosmic Rays 23rd, 3, 535-539.

Badhwar, G. D., and P. M. O'Neill (1994), Long term modulation of galactic cosmic radiation and its model for space exploration, Adv. Space Res., 14, 749-757, doi:10.1016/0273-1177(94)90537-1.

Badhwar, G. D., and P. M. O'Neill (1996), Galactic cosmic radiation model and its applications, Adv. Space Res., 17, 7-17, doi:10.1016/ 0273-1177(95)00507-B.

Beer, J., et al. (1990), Use of ${ }^{10} \mathrm{Be}$ in polar ice to trace the 11-year cycle of solar activity, Nature, 347, 164-166, doi:10.1038/347164a0.

Cini Castagnoli, G., et al. (1995), Evidence for enhanced ${ }^{10} \mathrm{Be}$ deposition in Mediterranean sediments 35 kyr BP, Geophys. Res. Lett., 22, 707-710, doi:10.1029/95GL00298.

Cini Castagnoli, G., G. Bonino, C. Taricco, and B. Lehman (1998), Cosmogenic isotopes and geomagnetic signals in a Mediterranean sea sediment at 35,000 y BP, Nuovo Cimento C, 21, 243-246.

Cucinotta, F. A., F. K. Manuel, J. Jones, G. Izsard, J. Murray, B. Djojonegro, and M. Wear (2001a), Space radiation and cataracts in astronauts, Radiat. Res., 156, 460-466, doi:10.1667/0033-7587(2001) 156[0460:SRACIA]2.0.CO;2.

Cucinotta, F. A., W. Schimmerling, J. W. Wilson, L. E. Peterson, G. D. Badhwar, P. Saganti, and J. F. Dicello (2001b), Space radiation cancer risks and uncertainties for Mars missions, Radiat. Res., 156, 682-688, doi:10.1667/0033-7587(2001)156[0682:SRCRAU]2.0.CO;2.

Ferreira, S. E., and M. S. Potgieter (2004), Long-term cosmic ray modulation in the heliosphere, Astrophys. J., 603, 744-752, doi:10.1086/ 381649.

Florinski, V., G. P. Zank, and N. V. Pogorelov (2003), Galactic cosmic ray transport in the global heliosphere, J. Geophys. Res., 108(A6), 1228, doi:10.1029/2002JA009695.

Forbush, S. E. (1954), World-wide cosmic-ray variations, 1937-1952, J. Geophys. Res., 59, 525-542, doi:10.1029/JZ059i004p00525.

Gleeson, L. J., and W. I. Axford (1968), Solar modulation of galactic cosmic rays, Astrophys. J., 154, 1011-1026, doi:10.1086/149822.

Heber, B., A. Kopp, J. Gieseler, R. Müller-Mellin, H. Fichtner, K. Scherer, M. S. Potgieter, and S. E. S. Ferreira (2009), Modulation of galactic cosmic ray protons and electrons during an unusual solar minimum, Astrophys. J., 699, 1956-1963, doi:10.1088/0004-637X/699/ $2 / 1956$.

Hess, V. (1912), Über beobachtungen der durchdringenden strahlung bei seiben freiballonfahrten, Phys. Z., 13, 1084-1091.

Hess, V. (1913), Über den ursprung der durchdringenden strahlung, Phys. Z., 14, 610-617.

Jokipii, J. R., E. H. Levy, and W. B. Hubbard (1977), Effects of particle drift on cosmic-ray transport. I: General properties, application to solar modulation, Astrophys. J., 213, 861-868, doi:10.1086/155218.

Masarik, J., and J. Beer (1999), Simulation of particle fluxes and cosmogenic nuclide production in the Earth's atmosphere, J. Geophys. Res., 104, 12,099-12,111, doi:10.1029/1998JD200091.

McComas, D. J., et al. (2009), Global observations of the interstellar interaction from the Interstellar Boundary Explorer (IBEX), Science, 326, 959-962, doi:10.1126/science.1180906.

McHargue, L. R., P. E. Damon, and D. J. Donahue (1995), Enhanced cosmic-ray production of ${ }^{10} \mathrm{Be}$ coincident with Mono Lake and Laschamp geomagnetic excursions, Geophys. Res. Lett., 22, 659662, doi:10.1029/95GL00169.

McHargue, L. R., D. J. Donahue, P. E. Damon, C. P. Sonett, D. Biddulph, and G. Burr (2000), Geomagnetic modulation of the late Pleistocene cosmic-ray flux as determined by ${ }^{10} \mathrm{Be}$ from Blake Outer Ridge marine sediments, Nucl. Instrum. Methods Phys. Res., Sect. B, 172, 555-561, doi:10.1016/S0168-583X(00)00092-6.

Millikan, R. A., and I. S. Bowen (1926), High frequency rays of cosmic origin I. Sounding balloon observations at extreme altitudes, Phys. Rev., 27(4), 353-361, doi:10.1103/PhysRev.27.353.

NASA Technical Standard (2007), NASA Space Flight Human System Standard, vol. 1, Crew Health, NASA-STD-3001, NASA, Washington, D. C.

O'Neill, P. M. (2006), Badhwar-O'Neill galactic cosmic ray model update based on Advanced Composition Explorer (ACE) energy spectra from 1997 to present, Adv. Space Res., 37, 1727-1733, doi:10.1016/j.asr.2005.02.001. 
Owens, M. J., N. A. Schwadron, N. U. Crooker, W. J. Hughes, and H. E. Spence (2007), Role of coronal mass ejections in the heliospheric Hale cycle, Geophys. Res. Lett., 34, L06104, doi:10.1029/ 2006GL028795.

Owens, M. J., C. N. Arge, N. U. Crooker, N. A. Schwadron, and T. S. Horbury (2008), Estimating total heliospheric magnetic flux from single-point in situ measurements, J. Geophys. Res., 113, A12103, doi:10.1029/2008JA013677.

Parker, E. N. (1965), Passage of energetic particles through interplanetary space, Planet. Space Sci., 13, 9-49, doi:10.1016/0032-0633(65) 90131-5.

Potgieter, M. S., and J. A. le Roux (1992), The simulated features of heliospheric cosmic-ray modulation with a time-dependent drift model. I-General effects of the changing neutral sheet over the period 1985-1990, Astrophys. J., 386, 336-346, doi:10.1086/171020.

Raisbeck, G. M., F. Yiou, D. Bourles, C. Lorius, J. Jouzel, and N. I. Barkov (1987), Evidence for two intervals of enhanced ${ }^{10} \mathrm{Be}$ deposition in Antarctic ice during the last glacial period, Nature, 326, 273-277, doi:10.1038/326273a0.

Richardson, J. D., J. C. Kasper, C. Wang, J. W. Belcher, and A. J. Lazarus (2008), Cool heliosheath plasma and deceleration of the upstream solar wind at the termination shock, Nature, 454, 63-66, doi:10.1038/nature07024.

Saganti, P. B., F. A. Cucinotta, J. W. Wilson, T. F. Cleghorn, and C. J. Zeitlin (2006), Model calculations of the particle spectrum of the galactic cosmic ray (GCR) environment: Assessment with ACE/ CRIS and MARIE measurements, Radiat. Meas., 41, 1152-1157, doi:10.1016/j.radmeas.2005.12.008.

Scherer, K., H. Fichtner, and O. Stawicki (2002), Shielded by the wind: The influence of the interstellar medium on the environment of the Earth, J. Atmos. Sol. Terr. Phys., 64, 795-804, doi:10.1016/S1364-6826 (02)00078-0.

Schwadron, N. A., M. Owens, and N. U. Crooker (2008), The heliospheric magnetic field over the Hale cycle, Astrophys. Space Sci. Trans., 4, 19-26.

Space Science Board (1973), HZE-Particle Effects in Manned Space Flight, Natl. Acad. of Sci., Washington, D. C.
Space Studies Board (1996), Radiation Hazards to Crews on Interplanetary Missions, Natl. Acad. of Sci., Washington, D. C.

Stone, E. C., A. C. Cummings, F. B. McDonald, B. C. Heikkila, N. Lal, and W. R. Webber (2005), Voyager 1 explores the termination shock region and the heliosheath beyond, Science, 309, 2017-2020, doi:10.1126/science.1117684.

Townsend, L. W., Y. M. Charara, N. Delauder, M. PourArsalan, J. A. Anderson, C. M. Fisher, H. E. Spence, N. A. Schwadron, M. J. Golightly, and F. A. Cucinotta (2010), Parameterizations of the linear energy transfer spectrum for the CRaTER instrument during the LRO mission, Space Weather, 8, S00E03, doi:10.1029/ 2009SW000526.

Urch, I. H., and L. J. Gleeson (1972), Galactic cosmic ray modulation from 1965 to 1970, Astrophys. Space Sci., 17, 426-446.

Wagner, G., J. Masarik, J. Beer, S. Baumgartner, D. Imboden, P. W. Kubik, H.-A. Synal, and M. Suter (2000), Reconstruction of the geomagnetic field between 20 and $60 \mathrm{kyr}$ BP from cosmogenic radionuclides in the GRIP ice core, Nucl. Instrum. Methods Phys. Res., Sect. B, 172, 597-604, doi:10.1016/S0168-583X(00)00285-8.

Wilson, J. W., L. W. Townsend, W. S. Schimmerling, G. S. Khandelwal, F. S. Khan, J. E. Nealy, F. A. Cucinotta, L. C. Simonsen, J. L. Shinn, and J. W. Norbury (1991), Transport methods and interactions for space radiations, NASA Tech. Rep., NASA-RP-1257, 615 pp.

Zank, G. P., and P. C. Frisch (1999), Consequences of a change in the galactic environment of the Sun, Astrophys. J., 518, 965-973, doi:10.1086/307320.

A. J. Boyd, K. Kozarev, and N. A. Schwadron, Department of Astronomy, Boston University, Boston, MA 02215, USA. (nathanas@ bu.edu)

M. Golightly and H. Spence, Institute for the Study of Earth Oceans, and Space, University of New Hampshire, Durham, NH 03824, USA.

M. Owens, Space Environment Physics Group, Department of Meteorology, University of Reading, Reading RG6 6BB, UK.

L. W. Townsend, Department of Nuclear Engineering, University of Tennessee, Knoxville, TN 37996, USA. 